\title{
Correlations in quantum spin chains and lattices
}

\author{
Document Version \\ Accepted author manuscript
}

Link to publication record in Manchester Research Explorer

\section{Citation for published version (APA):}

Bishop, RF., Parkinson, JB., \& Xian, Y. (1992). Correlations in quantum spin chains and lattices: A fully microscopic many-body approach. In TL. Ainsworth, CE. Campbell, BE. Clements, \& E. Krotscheck (Eds.), Recent Progress in Many-Body Theories, Vol. 3 (pp. 117-133). Plenum Publishing Corporation.

http://personalpages.manchester.ac.uk/staff/raymond.bishop/RFB_papers/[096] RPMBT_3(1992)117

\section{Published in:}

Recent Progress in Many-Body Theories, Vol. 3

\section{Citing this paper}

Please note that where the full-text provided on Manchester Research Explorer is the Author Accepted Manuscript or Proof version this may differ from the final Published version. If citing, it is advised that you check and use the publisher's definitive version.

\section{General rights}

Copyright and moral rights for the publications made accessible in the Research Explorer are retained by the authors and/or other copyright owners and it is a condition of accessing publications that users recognise and abide by the legal requirements associated with these rights.

\section{Takedown policy}

If you believe that this document breaches copyright please refer to the University of Manchester's Takedown Procedures [http://man.ac.uk/04Y6Bo] or contact uml.scholarlycommunications@manchester.ac.uk providing relevant details, so we can investigate your claim.

\section{OPEN ACCESS}




\section{Recent Progress in MANY-BODY THEORIES VOLUME 3}

Edited by

T. L. Ainsworth

Texas A\&M University

College Station, Texas

C. E. Campbell

University of Minnesota

Minneapolis, Minnesota

B. E. Clements

Texas A\&M University

College Station, Texas

and

E. Krotscheck

Texas A\&M University

College Station, Texas

Plenum Press - New York and London 
Proceedings of the Seventh International Conference on Recent Progress in Many-Body Theories, held August 26-31, 1991, in Minneapolis, Minnesota

Library of Congress Catalog Card Number 88-645051

ISBN 0-306-44246-9

(C) 1992 Plenum Press, New York

A Division of Plenum Publishing Corporation 233 Spring Street, New York, N.Y. 10013

All rights reserved

No part of this book may be reproduced, stored in a retrieval system, or transmitted in any form or by any means, electronic, mechanical, photocopying, microfilming, recording, or otherwise, without written permission from the Publisher

Printed in the United States of America 


\section{CORRELATIONS IN QUANTUM SPIN CHAINS AND LATTICES:}

\section{A FULLY MICROSCOPIC MANY-BODY APPROACH}

\section{R.F. Bishop, J.B. Parkinson and Yang Xian}

Department of Mathematics, UMIST

University of Manchester Institute of Science and Technology

P.O. Box 88, Manchester M60 1QD, England

\section{INTRODUCTION}

Low-dimensional quantum spin systems have long been studied within the framework of magnetism. Two recent developments have greatly added to their interest. These concern the Haldane conjecture for integral-spin one-dimensional (1D) antiferromagnets, and the link between two-dimensional (2D) antiferromagnetism and high-temperature superconductivity. In the latter case, among the various models proposed, the 2D Hubbard model ${ }^{2}$ is widely believed to contain the essential correlations of the active electrons in the ceramic oxide materials. Anderson ${ }^{3}$ proposed that the high- $T_{c}$ superconductivity might originate from the non-Fermi liquid behaviour away from half-filling. In the present context, the antiferromagnetic Heisenberg model can itself be derived from the Hubbard model at half filling, and this is believed to describe correctly the electronic properties of the high- $\mathrm{T}$. materials before doping. This point, first suggested by Anderson ${ }^{3}$ soon after their discovery, has now been supported by a large number of experiments. ${ }^{4}$

For the above reasons, it has become important to study these strongly-correlated models at a fundamental ab initio level. Although the recent literature has acquired enormous proportions, only a small fraction has been devoted to a fully microscopic approach. In particular, the coupled cluster method $(\mathrm{CCM})^{5}$ has proved to be one of the most powerful and universal high-precision techniques in quantum many-body theory. Its advantages include its capacity for systematic improvement via well-defined hierarchies of truncation schemes, and its automatic avoidance of unphysical divergences in the thermodynamic limit for infinite systems. The CCM has been well described elsewhere, ${ }^{5-13}$ and it has been applied to a wide range of physical systems. These include problems in nuclear physics, both for finite $9,44,15$ and infinite nuclear matter; ${ }^{16}$ atomic and molecular systems in quantum chemistry; $7,12,17$ and the electron gas; ${ }^{8,18}$ as well as such model field-theoretical systems as the quantum anharmonic oscillator treated as a single-mode bosonic field theory, ${ }^{19}$ and the relativistic $\Phi^{4}$ quantum field theory. ${ }^{20}$

In view of this impressive number of successful applications to diverse condensed-matter and field-theoretical systems, it seems timely to apply the CCM to quantum spin-lattice problems. We note that such models in general form very clean systems on which to deploy any technique of quantum many-body theory. They contain a nice admixture of exact results and unsolved problems 
which are both interesting in their own right and relevant to various physical systems. Furthermore, they are among the most quantum-mechanical of all systems, in that their behaviour is of ten quite different from that of their classical counterparts. Unexpected and counter-intuitive results abound.

Specifically, we mostly study here the spin- $\frac{1}{2}$ anisotropic antiferromagnets described by the $\mathrm{XXZ}$ (or Heisenberg-Ising) Hamiltonian, for both $1 \mathrm{D}$ chains and the $2 \mathrm{D}$ square lattice. These include the Heisenberg model as the special (isotropic) limit. We have already noted the perceived practical importance of the 2D model in connection with high-T superconductivity. Although to date the $2 \mathrm{D}$ models have not been exactly solved (and are not believed to be exactly integrable), their 1D XXZ-model counterparts are exactly soluble by the Bethe ansatz. ${ }^{21}$ Hence, we may compare our CCM results with exact results for such important ground-state properties as the energy, ${ }^{22}$ the staggered magnetization, ${ }^{23}$ and the asymptotic behaviour of the ${ }_{25}$ spin-spin correlation functions, ${ }^{24}$ as well as the excitation spectrum. ${ }^{25}$ We have briefly reviewed some of these exact results elsewhere. ${ }^{26}$

The XXZ model and various preliminary definitions are first described in Sec. 2, after which we discuss the CCM cluster decomposition of the ground ket state in Sec. 3, where we also outline several intuitively appealing and systematic approximation schemes. These are applied to calculate the ground-state (g.s.) energy of the $1 \mathrm{D}$ chain and the $2 \mathrm{D}$ square lattice respectively in Secs. 4 and 5. One of the key features of the CCM is that the Hilbert space has a biorthogonal structure, rather than the more usual orthogonal basis of alternative theories. Thus, the bra states defined in Sec. 6 are not manifestly Hermitian conjugate to the corresponding ket states at a given level of approximation, and must be calculated separately. With both bra and ket states defined we may then calculate any other ground-state property. As an example, we also calculate in Sec. 6 the staggered magnetization for both the $1 \mathrm{D}$ and $2 \mathrm{D}$ cases. The corresponding excitation spectra are discussed in Sec. 7. For particular approximation schemes which incorporate long-range correlations we find very strong evidence in both $1 D$ and $2 \mathrm{D}$ for quantum phase transitions at critical values of the anisotropy parameter, although with qualitatively different features in the two cases. We briefly discuss extensions of our method to systems of spins with $s>\frac{1}{2}$ in Sec. 8, and then summarize in Sec. 9.

\section{THE MODEL AND PRELIMINARIES}

We consider an even number $N$ of quantum spins $\vec{s}_{k} \equiv\left\{s_{k}^{a} ; a=x, y, z\right\}$ placed on the sites $\{k\}$ of a specified regular lattice in $d$ dimensions whose coordination number is $z$, with periodic boundary conditions. They obey the usual SU(2) algebra,

$$
\left[s_{k}^{a}, s_{m}^{b}\right]=i \delta_{k m} \varepsilon_{a b c} s_{k}^{c},
$$

where $\varepsilon_{\text {abc }}$ is the usual antisymmetric unit tensor and the summation convention is employed. We shall mostly be interested in the case where the spin quantum number $s=\frac{1}{2}$, with $\vec{s}_{k}^{2}=s(s+1)$, and where $N \rightarrow \infty$. Furthermore, we consider only bipartite lattices (i.e., ones in which the lattice can be decomposed into two equivalent sublattices such that all $z$ nearest neighbours of a site on one sublattice belong to the other one), with $\frac{1}{2} \mathrm{~N}$ spins on each sublattice.

The XXZ-model Hamiltonian,

$$
\mathrm{H}=\frac{1}{2} \sum\left(\mathrm{s}_{\ell}^{\mathrm{x}_{\ell+\rho}} \mathrm{s}_{\ell+\rho}+\mathrm{s}_{\ell}^{\mathrm{y}} \mathrm{s}_{\ell+\rho}^{\mathrm{y}}+\Delta \mathrm{s}_{\ell}^{\mathrm{z}} \mathrm{s}_{\ell+\rho}^{\mathrm{z}}\right),
$$


is a model of nearest-neighbour anisotropic interactions between the localized spins, where the index $\ell$ runs over all $\mathrm{N}$ lattice sites and the index $\rho$ over all $\mathrm{z}$ nearest-neighbour lattice vectors. We take both the lattice spacing and the antiferromagnetic coupling constant to be unity. The Ising, Heisenberg, and (XY or) planar models correspond respectively to the three values $\Delta \rightarrow \infty$, $\Delta=1$, and $\Delta=0$ of the anisotropy parameter $\Delta$. The Hamiltonian of Eg. (2) commutes with the $z$-component of the total spin operator, $s_{z}^{\text {total }} \equiv \sum_{\ell} s_{\ell^{\prime}}^{z}$ which is hence a good quantum number. We are especially interested in those values of $\Delta$ for which the ground state is antiferromagnetic and hence lies in the subspace $s_{z}^{\text {total }}=0$.

For the spin- $\frac{1}{2}$ models of primary interest we replace the spin operators by the Pauli operators, $\sigma^{a} \equiv 2 s^{a}$. Furthermore, since we are interested in antiferromagnetic states, the Néel state (in which the spins are perfectly aligned in opposing directions on the sublattices, say along the $\pm z$-axes) is bound to play a special role. It is thus very convenient to choose from the outset different coordinate axes on the two sublattices, so that all of the spins in the Neel state point in the same named direction (say along the -z-axis or in the "down" direction). This is equivalent to performing a purely notional rotation of $180^{\circ}$ (about the y-axis, say) of the spins on the "up" sublattice, leading to the transformation $s^{x} \rightarrow-s^{x}, s^{y} \rightarrow s^{y}, s^{z} \rightarrow-s^{z}$ on this sublattice. We may thus equivalently define raising and lowering operators by,

$$
\sigma_{i}^{ \pm} \equiv \frac{1}{2}\left(\sigma_{i}^{x} \pm i \sigma_{i}^{y}\right) ; \quad \sigma_{j}^{ \pm} \equiv \frac{1}{2}\left(-\sigma_{j}^{x} \pm i \sigma_{j}^{y}\right)
$$

for sites $i$ and $j$ on the "down" and "up" sublattices respectively. This sublattice rotation leaves the commutation relations invariant, namely,

$$
\left[\sigma_{\mathrm{k}}^{+}, \sigma_{\mathrm{m}}^{-}\right]=\sigma_{\mathrm{k}}^{\mathrm{z}} \delta_{\mathrm{km}} ;\left[\sigma_{\mathrm{k}}^{\mathrm{z}}, \sigma_{\mathrm{m}}^{ \pm}\right]= \pm 2 \sigma_{\mathrm{k}}^{ \pm} \delta_{\mathrm{km}},
$$

for sites $k$ and $m$ on either sublattice. In this notation the Néel state $|\Phi\rangle$ has all spins pointing "down", so that $\sigma_{k}^{-}|\Phi\rangle=0$ for all $k$, and $\sigma_{k}^{+}|\Phi\rangle$ is a state with the $k^{\text {th }}$ spin reversed with respect to the Néel state. In the Néel basis used henceforth, the Hamiltonian becomes,

$$
\mathrm{H}=-\frac{1}{4} \sum_{\ell, \rho}\left(\sigma_{\ell}^{+} \sigma_{\ell+\rho}^{+}+\sigma_{\ell}^{-} \sigma_{\ell+\rho}^{-}+\frac{1}{2} \Delta \sigma_{\ell}^{z} \sigma_{\ell+\rho}^{z}\right)
$$

\section{CCM PARAMETRIZATION OF THE GROUND KET STATE}

The Neel state $|\Phi\rangle$ does not represent the g.s. of the XXZ-model Hamiltonian except in the Ising limit $\Delta \rightarrow \infty$. For finite values of $\Delta$, quantum fluctuations play a significant role, especially for low-dimensional lattices and for small values of the spin. Equivalently, we may say that the exact ground ket state $|\Psi\rangle$, where

$$
\mathrm{H}|\Psi\rangle=\mathrm{E}_{g}|\Psi\rangle,
$$

has (multi-spin) fluctuations or correlations not present in $|\Phi\rangle$. These are incorporated in the CCM via a correlation operator S,

$$
|\Psi\rangle=e^{S}|\Phi\rangle \text {, }
$$

which was perhaps first employed for spin-lattice systems by ${ }_{3}$ Roger and Hetherington, ${ }^{27}$ who were primarily interested in the solid phases of ${ }^{3} \mathrm{He}$. 
As usual in the $C C M,{ }^{5,8-11}$ the operator $S$ is constructed from products of creation operators with respect to $|\Phi\rangle$, namely in this case the Néel-basis operators $\left\{\sigma_{\mathbf{k}}^{+}\right\}$defined in Eq. (3). We thus put

$$
S=\sum_{m=1}^{N} S
$$

where $S_{m}$ creates a linear combination of configurations with $m$ spins flipped with respect to the Néel state. Since the g.s. $|\Psi\rangle$ of the antiferromagnetic models lies in the subspace $\mathbf{s}_{\mathbf{z}}^{\text {total }}=0$, the correlation operator $\mathrm{S}$ should contain only those partitions $S_{m}$ with $\mathrm{m}$ even, and with $\frac{1}{2} \mathrm{~m}$ creation operators on each sublattice. Henceforth, we use the indices $i$ or $\left\{i_{n}\right\}$ and $j$ or $\left\{j_{n}\right\}$ to indicate sites on the two respective sublattices. We may then decompose the nonzero g.s. cluster correlation operators in the form,

$$
S_{2 n}=\frac{1}{(n !)^{2}} \sum_{i_{1}, \ldots, i_{n} j_{1}, \ldots, j_{n}} S_{i_{1} \ldots i_{n} ; j_{1} \ldots j_{n}} \sigma_{i_{1}}^{+} \ldots \sigma_{i_{n}}^{+} \sigma_{j_{1}}^{+} \ldots \sigma_{j_{n}}^{+},
$$

where any coefficient $S_{i_{1} \ldots i_{n} ; j_{1} \ldots j_{n}}$ with any repeated index may be taken as

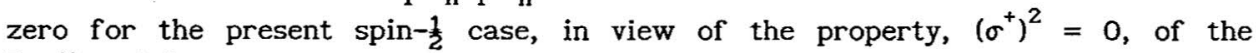
Pauli matrices.

The equations which determine the cluster configuration coefficients $\left\{S_{i_{1} \ldots i} ; j_{1} \ldots j, j_{n}\right\}$ are now found in the usual CCM way by first rewriting the g.s. Schrödinger equation of Eqs. (6) and (7) as,

$$
e^{-S_{H e}} S|\Phi\rangle=E_{g}|\Phi\rangle \text {. }
$$

We note that, as usual, the similarity-transformed Hamiltonian, $\mathrm{e}^{-\mathrm{S}_{\mathrm{He}}} \mathrm{S}$, can be expanded in terms of the well-known nested commutator series. By making use of the commutation relations of Eq. (4), it is easy to see that this otherwise infinite series now, however, terminates after terms of fourth order in $\mathrm{S}$ for the Hamiltonian of Eq. (5). Secondly, the transformed Eq. (10) is then projected in turn with the model Neel state $\langle\Phi|$ and with the Hermitian adjoints of each of the configurations contained in (either the exact or the particular approximation being used for) the cluster operator S. We thereby obtain respectively the exact relation,

$$
\frac{E_{g}}{N}=-\frac{z}{8}\left(\Delta+2 b_{1}\right)
$$

for the g.s. energy, where $\mathbf{z}$ is the lattice coordination number and $S_{i j i+\rho} \equiv b_{1}$ is the nearest-neighbour two-spin-flip coefficient (which is independent of both $\mathrm{i}$ and $\rho$ by translational invariance and by the symmetries. of the lattice under rotations and reflections, respectively), plus a set of coupled nonlinear algebraic equations for the various cluster configuration coefficients.

Approximations within the CCM now come from truncating the sum over all possible cluster-configuration coefficients in Eqs. (8) and (9) to some (finite or infinite) subset. Several systematic schemes have been investigated by us, ${ }^{26,28}$ and we briefly review some of them below. The hierarchy most commonly employed in other CCM applications is the so-called SUBn scheme, in which one keeps only up to $n$-spin correlations by truncating the sum in Eq. (8) by the replacement $\mathrm{N} \rightarrow \mathrm{n}$, 


$$
S \rightarrow S_{\text {SUBn }}=\sum_{\ell=1}^{n / 2} S_{2 \ell}
$$

and in the present case $\mathrm{n}$ is an even integer. For example, in SUB4 approximation we now have two coupled sets of equations for the 2-spin-flip and 4-spin-flip coefficients, $\left\{S_{i ; j^{\prime}}\right\}$ and $\left\{S_{i 1^{\prime} ; j^{\prime}}\right\}$ respectively. These are obtained as described above by first replacing $S \rightarrow S_{\text {SuB4 }}$, and then taking the respective overlaps of Eq. (10) with the states $\sigma_{i}^{+} \sigma_{j}^{+}|\Phi\rangle$ and $\sigma_{i}^{+} \sigma_{i}^{+}, \sigma_{j}^{+} \sigma_{j}^{+},|\Phi\rangle$. We quote only the final result for the former (2-spin-flip) case,

$$
\begin{aligned}
& \sum_{\rho}\left[\left(1+2 \Delta S_{i ; i+\rho}+2 S_{i ; i+\rho}^{2}\right) \delta_{i+\rho, j}-2\left(\Delta+2 S_{i ; i+\rho}\right) S_{i ; j}\right. \\
& \left.+\sum_{i}\left(S_{i ; i^{\prime}+\rho^{\prime} i^{\prime} ; j}+S_{i i^{\prime} ; i^{\prime}+\rho, j}\right)\right]=0 .
\end{aligned}
$$

Equation (13) is valid for a spin- $\frac{1}{2}$ bipartite lattice of arbitrary dimensionality. We note also that Eq. (13), like Eq. (11), is actually exact, whereas its SUB4 counterpart for the 4-spin-flip coefficients, which we do not quote here, is only approximate since the coupling to the 6-spin-flip terms is neglected at this level.

The lowest (SUB2) approximation in the SUBn scheme is of special interest, as we see below. By using the translational invariance properties, we may write the 2-spin-flip coefficients as $S_{1 ; i+r} \equiv b_{r}$, where $r$ is a lattice vector connecting sites on opposite sublattices. The correlation operator $\mathrm{S}$ thus has the SUB2 approximant,

$$
S \rightarrow S_{\text {SUB2 }}=\sum_{i} \sum_{r} b_{r} \sigma_{i}^{+} \sigma_{i+r}^{+}=\frac{1}{2} \sum_{\ell} \sum_{r} b_{r} \sigma_{\ell}^{+} \sigma_{\ell+r}^{+},
$$

where the indices $\{i\}$ run over one sublattice only, while $\{\ell\}$ run over the entire lattice, as above. The SUB2 equations for the coefficients $\left\{b_{r}\right\}$ can easily be obtained from Eq. (13) by setting the 4-spin-flip coefficients to zero. We obtain

$$
\begin{aligned}
& 2 z K_{r}-\left(1+2 \Delta b_{1}+2 b_{1}^{2}\right) \sum_{\rho} \delta_{r \rho}-\sum_{r} \sum_{\rho} b_{r-r^{\prime}+\rho} b_{r^{\prime}}=0 ; \\
& K \equiv \Delta+2 b_{1} .
\end{aligned}
$$

As explained elsewhere, ${ }^{26,28}$ Eq. (15) can be solved exactly in arbitrary dimensionality for any bipartite hyper-cubic lattice, by Fourier transform techniques.

A second quite different set of approximations, called the LSUBn scheme, is based on the localized nature of the interactions. At a given LSUBn level we retain only those configurations in the correlation operator $S$ which contain (any number up to $\mathbf{n}$ of) spin-flips, with respect to the Néel state, over a locale or localized region of $n$ contiguous sites on the lattice, and which are compatible with the restriction $s_{z}^{\text {total }}=0$. Clearly, the lowest member of this sequence, LSUB2, retains only the single configuration of two spins flipped on nearest-neighbour sites, and which has the coefficient $b_{1}$. By setting to zero all coefficients ${\left\{b_{r}\right\}}_{r}$ in Eq. (15) other than $b_{\rho} \equiv b_{1}$, we 
find the simple quadratic form for the LSUB2 equation,

$$
\begin{aligned}
& 3 b_{1}^{2}+2 \Delta b_{1}-1=0 ; \quad 1 D \text { chain, } \\
& 5 b_{1}^{2}+6 \Delta b_{1}-1=0 ; \quad 2 D \text { square lattice. }
\end{aligned}
$$

A third approximation scheme, denoted as SUBn+LSUBm (with $m>n$ ), is a rather self-evident combination of the previous two schemes.

We have also investigated a fourth (PSUBn) scheme ${ }^{26}$ based on "plaquettes" of flipped spins. It was motivated both by the kink structures of spin systems in $1 \mathrm{D}$ and the domain-wall structures in higher dimensions. We do not consider it further in the present work.

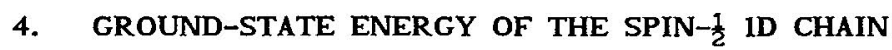

In 1D, the LSUBn sequence of approximations is defined on "locales" comprising $\mathbf{n}$ adjacent sites on the chain. For example, in LSUB4 and LSUB5 approximations we have,

$$
\begin{aligned}
& S \rightarrow S_{\text {LSUB4 }}=\sum_{\ell=1}^{N}\left(b_{1} \sigma_{\ell}^{+} \sigma_{\ell+1}^{+}+b_{3} \sigma_{\ell}^{+} \sigma_{\ell+3}^{+}+g_{4} \sigma_{\ell}^{+} \sigma_{\ell+1}^{+} \sigma_{\ell+2}^{+} \sigma_{l+3}^{+}\right) ; \\
& S \rightarrow S_{\text {LSUB5 }}=S_{\text {LSUB } 4}+\sum_{\ell=1}^{N} h_{5} \sigma_{\ell}^{+} \sigma_{\ell+1}^{+} \sigma_{\ell+3}^{+} \sigma_{\ell+4}^{+} ;
\end{aligned}
$$

while the LSUB6 approximation retains three extra independent (under the symmetries) 4-spin-flip configurations, plus a single 6-spin-flip configuration; etc. Results for the g.s. energy, calculated from Eq. (11), using this sequence, are shown in Fig. 1 and Table 1 . We note that even the LSUB2 expression from Eq. (17a), $\mathrm{E}_{\mathrm{g}} / \mathrm{N}=-\left[\Delta+2\left(\Delta^{2}+3\right)^{\frac{1}{2}}\right] / 12$, gives results of impressive accuracy when compared with the exactly known results ${ }^{22}$ for this case. It also gives the correct leading asymptotic form for large $\Delta$,

$$
\frac{\mathrm{E}}{\mathrm{N}} \underset{\Delta \rightarrow \infty}{\longrightarrow}-\frac{1}{4}\left(\Delta+\Delta^{-1}\right) ; \quad \text { 1D chain. }
$$

For the Heisenberg model $(\Delta=1)$, the LSUB2 result, $E_{g} N=-5 / 12 \approx-0.417$, may be compared with both the exact result of -0.443 and with the result of -0.428 obtained $^{29}$ by actually minimizing the expectation value of the Hamiltonian over all wavefunctions of the LSUB2 type (i.e., with respect to the parameter $\left.b_{1}\right)$.

The SUB2 approximation in 1D retains all of the 2-spin-flip configurations, with coefficients $S_{i ; i+r} \equiv b_{r}=b_{-r}$, where $r$ is an odd integer. The $(\mathrm{N} \rightarrow \infty)$ solution of the corresponding SUB2 equations of Eq. (15) in this case is given by,

$$
\begin{aligned}
& \mathrm{b}_{\mathrm{r}}=\frac{\mathrm{K}}{\pi} \int_{0}^{\pi} \mathrm{dq} \frac{\cos (\mathrm{rq})}{\cos (\mathrm{q})}\left[1-\sqrt{1-\mathrm{k}^{2} \cos ^{2} \mathrm{q}}\right] ; \\
& \mathrm{k}^{2} \equiv\left(1+2 \Delta \mathrm{b}_{1}+2 \mathrm{~b}_{1}^{2}\right) / \mathrm{K}^{2} .
\end{aligned}
$$

Putting $r=1$ in Eq. (20) leads to a self-consistent equation for $b_{1}$, which may be solved numerically. In this way we obtain the results for the g.s. 


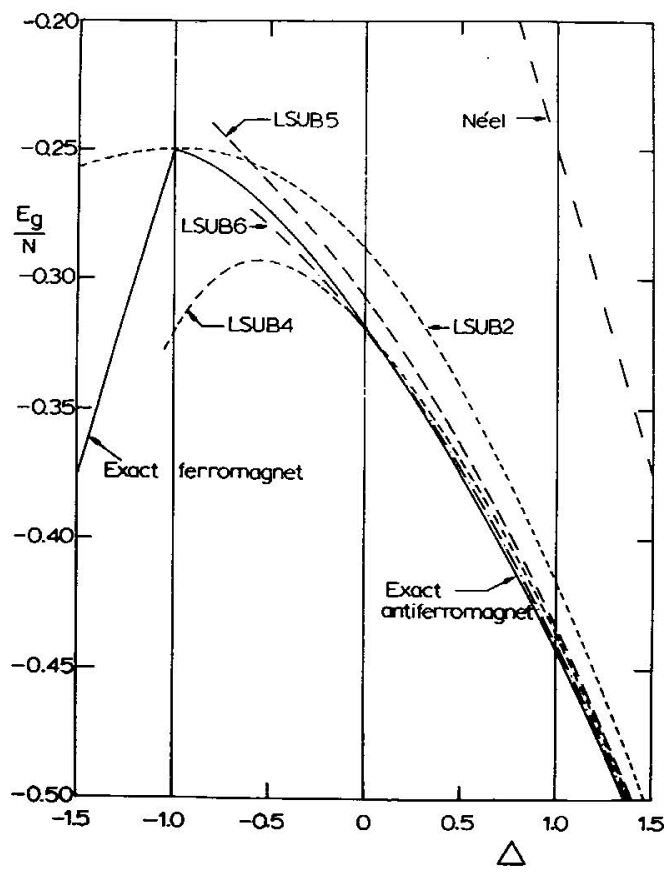

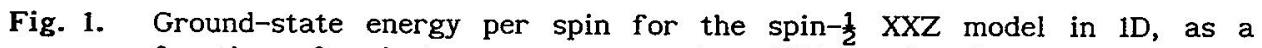
function of anisotropy parameter $\triangle$. CCM results for various LSUBn approximations are compared with the uncorrelated (classical) Neel result, $\mathrm{E}_{\mathrm{g}} / \mathrm{N}=-\frac{1}{4} \Delta$, and exact results.

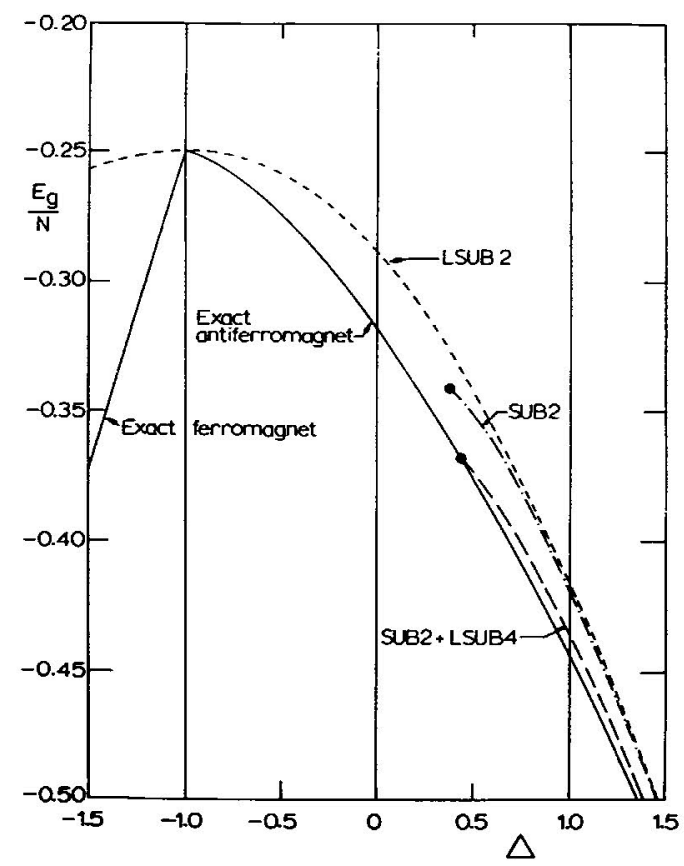

Fig. 2. As in Fig. 1, but showing CCM results in SUB2 and SUB2+LSUB4 approximations, which include long-range correlations. Their corresponding terminating points are clearly indicated. 
Table 1. The g.s. energy per spin, $E_{g} / N$, for the infinite $(N \rightarrow \infty)$, spin- $\frac{1}{2}$, 1D $\mathrm{XXZ}$-model chain, for various values of the anisotropy parameter $\Delta$.

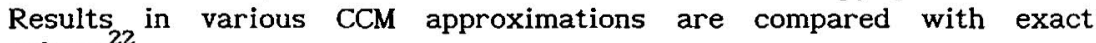
values. 22

\begin{tabular}{cccccccc}
\hline & \multicolumn{7}{c}{$E_{\mathbf{g}} / \mathrm{N}$} \\
\cline { 2 - 8 } & LSUB2 & LSUB4 & LSUB5 & LSUB6 & SUB2 & $\begin{array}{c}\text { SUB2+ } \\
\text { LSUB4 }\end{array}$ & Exact \\
\hline-0.5 & -0.2588 & -0.2932 & -0.2638 & -0.2797 & - & - & -0.2745 \\
0.0 & -0.2887 & -0.3193 & -0.3078 & -0.3198 & -- & -- & -0.3183 \\
0.5 & -0.3421 & -0.3692 & -0.3638 & -0.3730 & -0.3506 & -0.3727 & -0.3750 \\
1.0 & -0.4167 & -0.4363 & -0.4339 & -0.4400 & -0.4186 & -0.4366 & -0.4432 \\
1.5 & -0.5069 & -0.5195 & -0.5185 & -0.5218 & -0.5075 & -0.5196 & -0.5234 \\
2.0 & -0.6076 & -0.6155 & -0.6151 & -0.6167 & -0.6079 & -0.6155 & -0.6172 \\
\hline
\end{tabular}

energy shown in Table 1 and Fig. 2. We note that the SUB2 energies are surprisingly close to the much simpler LSUB2 estimates for most values of $\Delta$. For example, at $\Delta=1$ the SUB2 result is $\mathrm{E}_{\mathbf{g}} / \mathrm{N} \approx-0.419$. Its asymptotic behaviour also agrees with the exact result of Eq. (19).

The most interesting feature of the SUB2 results is that a real solution for $b_{1}$ ceases to exist when $k_{>}>k_{c}=1$, or equivalently when $\Delta<\Delta_{c} \approx 0.373$. The absence of a solution for $\Delta<\Delta_{c}$ is a clear signal of a possible phase transition, even though the value of $\Delta_{c}$ is not very close to the exact critical value of 1 at which it is known that the spin- $\frac{1}{2} \mathrm{XXZ}$-model chain undergoes a phase transition. Thus, for $\Delta>1$ the 1D chain has an ordered, Ising-like, antiferromagnetic phase with exponentially-decaying spin-spin correlation functions at large distances, and a spin-wave excitation spectrum with a finite gap. Conversely, for $|\Delta|<1$ it has a "critical" phase with correlations which decay algebraically to zero at large distances, and a gapless excitation spectrum.

Clearly, it is desirable to have additional evidence that the breakdown of the SUB2 solution for $\Delta<\Delta_{c}$ is not merely a mathematical artifice. To this end we calculate the corresponding staggered magnetization and the excitation spectrum below in Secs. 6 and 7. For the moment we note only the highly suggestive changeover at $\Delta=\Delta_{c}$ in the long-range behaviour of the 2-spin-flip coefficients $\left\{b_{\mathbf{r}}\right\}$ from exponential to power-law decay,

$$
b_{r} \underset{r \rightarrow \infty}{\longrightarrow}\left\{\begin{array}{l}
a / \ell^{r}, \Delta>\Delta \\
b / r^{2}, \Delta=\Delta_{c}^{c} ;
\end{array} ;\right.
$$

where $\mathrm{b}$ is a constant, and $\mathrm{a}$ and $\ell(>1)$ are functions only of $\Delta$.

Finally, we have also considered the SUB2+LSUB4 approximation, since one expects that such a scheme which retains the long-range correlations of the SUB2 approximation, but which also includes the extra short-range 4-spin-flip contribution which seems to be important for increasing the accuracy of the g.s. energy estimation, could continue to show a phase transition. The quantitative results shown in Table 1 are gratifying. Furthermore, the SUB2 critical point is not only not destroyed, but the SUB2+LSUB4 value, $\Delta_{c} \approx 0.436$ is even slightly closer to the expected value of 1 . 
Table 2. The g.s. energy per spin, $E_{g} / N$, for the spin- $\frac{1}{2}$ Heisenberg model on an infinite $(\mathrm{N} \rightarrow \infty)$ 2D square lattice. Results in various CCM schemes are compared with those from linear spin-wave theory (SWT) ${ }^{32}$ and from Green's function Monte Carlo (GFMC) calculations.

\begin{tabular}{lllllll}
\hline LSUB2 & SUB2 & LSUB4' & SUB2 $^{2}{ }_{4}^{\mathrm{a}}$ & LSUB4 & SWT & GFMC \\
\hline-0.648 & -0.651 & -0.653 & -0.656 & -0.664 & -0.658 & -0.669 \\
\hline
\end{tabular}

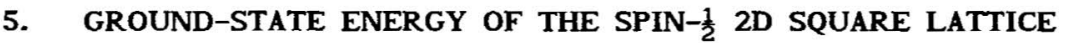

We have also performed the counterparts of most of the above calculations on the $1 D$ chain for the $2 \mathrm{D}$ square lattice, where very few exact results are known. The LSUBn approximation schemes in particular are now more complicated, however, due to the increased number of spin-flip configurations. For example, we show in Fig. 3 the seven independent configurations that are included in the LSUB4 approximation, together with their respective coefficients in the expansion of the cluster operator $\mathrm{S}$, and two possible measures, $w$ and $d$ of their relative importance. The first is simply the number $w$ of "wrong" bonds, with respect to the Neel state, of a given configuration. Classically, the breaking of each bond in the antiferromagnetic regime costs energy, and configurations with the smallest values of $\mathrm{w}$ hence seem likely to be most important. Secondly, we also define a weight parameter, $d$, as the length of the "domain boundary" of a given configuration of flipped spins. It is defined as the number of lattice bonds crossed by the shortest-path circuit (shown in Fig. 3 by the dashed lines) which encloses all of the flipped spins.

Taken together, the two weights $\mathrm{w}$ and $\mathrm{d}$ indicate a relative order of decreasing importance of the seven LSUB4 configurations as: $b_{1} ; g_{4}^{a} ; \quad\left(b_{3}^{a}, b_{3}^{b}\right)$; $\left(g_{4}^{b}, g_{4}^{c}, g_{4}^{d}\right)$. With this in mind, we have also performed, apart from full (LSUB2 and) LSUB4 calculations, the subapproximation which keeps only the two most highly weighted coefficients, $b_{1}$ and $g_{4}^{a}$. It is denoted as the LSUB4' scheme. Finally, we have also performed a full SUB2 and a partial SUB2+LSUB4 calculation, namely SUB $2+g_{4}^{2}$. They again yield critical values $\Delta_{c}$, for values of $\Delta$ below which the corresponding (real) solutions do not exist. For the two schemes SUB2 and SUB2 $+g_{4}$, the respective values for the $2 \mathrm{D}$ square lattice are $\Delta_{c} \approx 0.799$ and 0.819 , both considerably closer to the classical value of 1 than in $1 \mathrm{D}$.

Other results are shown in Fig. 4, and in Table 2 for the Heisenberg model. Since no exact $2 \mathrm{D}$ results are known we make comparison with the best available Green's function Monte Carlo (GFMC) results ${ }^{30}$ for $\Delta=1$, and with other recent Monte Carlo results. ${ }^{31}$ We also compare with the corresponding result of Oguchi from Anderson's approximate spin-wave theory (SWT), ${ }^{32}$ in which the correction to the classical Néel value is calculated to first order in inverse powers of the spin quantum number $s$ (where $s=\frac{1}{2}$ here). Our results again agree well with the essentially exact GFMC results.

\section{CCM PARAMETRIZATION OF THE GROUND BRA STATE; STAGGERED MAGNETIZATION}

For reasons that have been well documented elsewhere, ${ }^{10,11}$ the CCM 


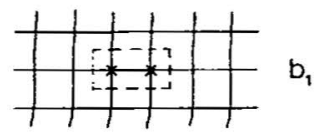

$w=6, \quad d=6$
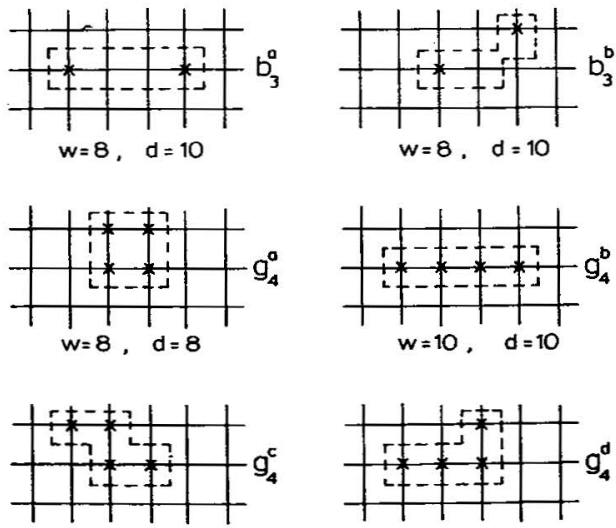

$w=10, d=10$

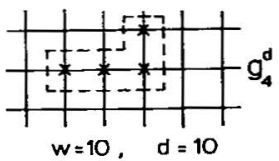

Fig. 3. The seven independent configurations retained in the LSUB4 scheme for the spin- $\frac{1}{2} 2 \mathrm{D}$ square lattice, together with their weights $\mathrm{w}$ and $d$, defined in the text. Crosses indicate the flipped spins, and the dashed lines delineate the corresponding "locale".

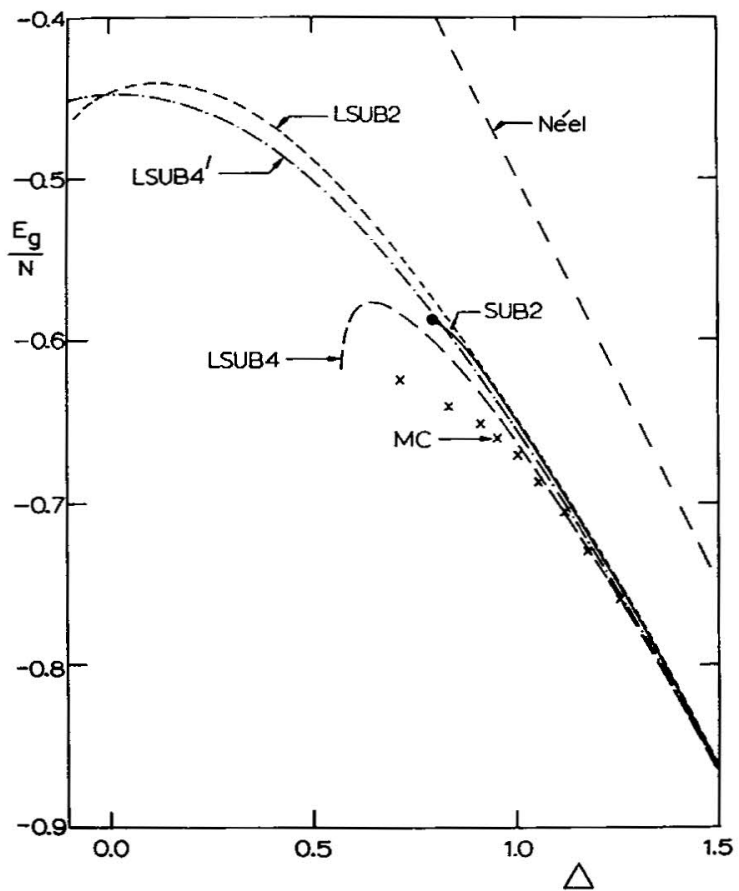

Fig. 4. Ground-state energy per spin for the spin- $\frac{1}{2} \mathrm{XXZ}$ model on the 2D square lattice, as a function of anisotropy parameter $\Delta$, showing CCM results for various approximations described in the text, and Monte Carlo (MC) results of Ref. 31 (indicated by crosses). The terminating point of the SUB2 scheme is indicated. 
parametrizes bra states in a form that is not the explicit Hermitian adjoint of their corresponding ket states. One basic reason is to preserve consistency with the important Hellmann-Feynman theorem at any given level of truncation. A disadvantage is that the CCM estimates for the g.s. energy are therefore not variational upper bounds (as would be the case in the simpler configuration-interaction method, for example, although this method is not size-extensive and hence not useful for present purposes). However, one also gains the considerable advantage that the expectation value of an arbitrary operator is a finite-order functional of the various configuration amplitudes (by contrast with the case when the bra states are taken as the Hermitian adjoints of the CCM ket states already defined).

In the so-called "normal" version of the CCM which we employ here (to contrast it with the more recent and even more powerful "extended" version ${ }^{10,11}$ ), the g.s. bra wavefunction corresponding to the ket state $|\Psi\rangle$ is parametrized as,

$$
\langle\tilde{\Psi}|=\langle\Phi| \tilde{\mathrm{S}} \mathrm{e}^{-\mathrm{S}},
$$

where the new linear correlation operator $\tilde{\mathrm{S}}$ is constructed wholly from products of destruction operators $\left\{\sigma_{\ell}^{-}\right\}$, apart from a constant term chosen to be unity to preserve the normalization, $\langle\tilde{\Psi} \mid \Psi\rangle=\langle\Phi|\tilde{S}| \Phi\rangle=1$. Otherwise, its construction is exactly as in Eqs. (8) and (9), except for the replacement $\left\{\sigma_{\ell}^{+}\right\} \rightarrow\left\{\sigma_{\ell}^{-}\right\}$. For example, the analogue of Eq. (14) in SUB2 approximation is,

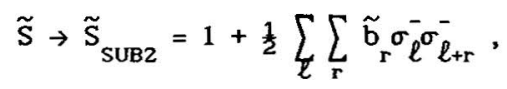

where the indices $\{\ell\}$ and $\{r\}$ have the same meaning as before. The equations for the bra-state configuration coefficients retained in any particular approximation are now obtained exactly as before by taking the overlap of the Schrödinger equation,

$$
\langle\tilde{\Psi}| \mathrm{H}=\mathrm{E}_{\mathrm{g}}\langle\tilde{\Psi}|,
$$

with each of the ket-state wavefunctions corresponding to the particular configurations retained. For example, in SUB2 approximation, by taking the overlap with the states $\sigma_{\ell}^{+} \sigma_{\ell+r}^{+}|\Phi\rangle$, we find,

$$
2 z K \tilde{b}_{r}-\left(1+2 K \tilde{b}_{1}-4 \sum_{r^{\prime}}, \tilde{b}_{r^{\prime}} b_{r^{\prime}}\right) \sum_{\rho} \delta_{r \rho}-2 \sum_{r} \sum_{\rho} b_{r-r^{\prime}+\rho^{\prime}} \tilde{b}_{r^{\prime}}=0,
$$
as the bra-state equivalent of the SUB2 ket-state equation (15). This
equation has also been solved by Fourier transform techniques. ${ }^{26,28}$ Similarly, the LSUB2 approximation, which now retains only the two coefficients $\left(b_{1}, \tilde{b}_{1}\right)$, is trivially solved.

We may now calculate the g.s. expectation value of an arbitrary operator in any particular CCM approximation. As an example we quote results for the staggered magnetization defined as,

$$
M^{z} \equiv-\frac{2}{N} \sum_{1}\left\langle\sigma_{i}^{z}\right\rangle=-\frac{2}{N} \sum_{i}\left\langle\Phi\left|\tilde{S} e^{-S} \sigma_{i}^{z} e^{S}\right| \Phi\right\rangle,
$$

where the summation runs over one sublattice only. If Eq. (27) is evaluated within the SUB2 approximation, we find for a bipartite lattice of arbitrary dimensionality, 


$$
M^{z}=1-2 \sum_{r} \tilde{b}_{r_{r}}
$$

which reduces to the corresponding LSUB2 expressions,

$$
M^{z}=1-2 z \tilde{b}_{1} b_{1}= \begin{cases}{\left[1+2 \Delta\left(\Delta^{2}+3\right)^{-\frac{1}{2}}\right] / 3,} & \text { 1D chain } ; \\ {\left[1+12 \Delta\left(9 \Delta^{2}+5\right)^{-\frac{1}{2}}\right] / 5,} & \text { 2D square lattice }\end{cases}
$$

Both the LSUB2 and full SUB2 results give the correct leading $O\left(\Delta^{-2}\right)$ correction to the Ising value of 1 for large values of $\Delta$.

The LSUB2 and SUB2 values of $\mathrm{M}^{2}$ as a function of $\Delta$ are shown in Fig. 5 for the spin- $\frac{1}{2}$ XXZ model on both the $1 \mathrm{D}$ chain and the 2D square lattice. In both cases we compare with results of Anderson's linear (lowest-order) spin-wave theory (SWT) ${ }^{32}$ In 1D we also compare with the exact results of Baxter, ${ }^{23}$ and in $2 \mathrm{D}$ with Monte Carlo results. ${ }^{30,31}$ Our main finding is that the behaviour of $\mathrm{M}^{\mathrm{z}}$ near $\Delta_{\mathrm{c}}$ in our SUB2 calculation now lends strong evidence
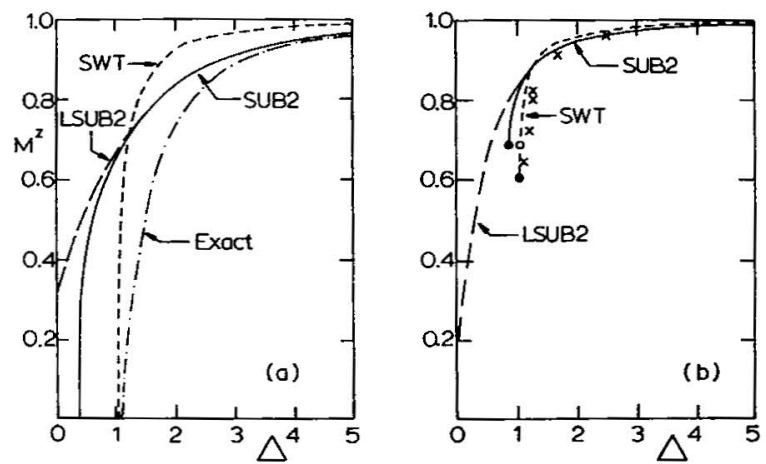

Fig. 5. Staggered magnetization $M^{2}$ for the spin- $\frac{1}{2}$ XXZ model: (a) in $1 D$, and (b) on the $2 \mathrm{D}$ square lattice, as functions of $\triangle$. Our LSUB2 and SUB2 results are compared with those from linear spin-wave theory (SWT). ${ }^{3}$ In $1 D$, exact results ${ }^{23}$ are shown. In $2 \mathrm{D}$, we show the Monte Carlo (MC) results of Ref. 31 (crosses) and of Carlson ${ }^{30}$ (circle).

for a phase transition at this point in our approximate calculations. Furthermore, we find that as $\Delta \rightarrow \Delta_{c}, M^{z} \rightarrow 0$ in $1 D$, whereas $M^{z} \rightarrow M_{c}^{z} \approx 0.682$ in 2D. This result is important since we believe that the critical points at $\Delta=$ $\Delta_{c}$ in our calculations approximate those at $\Delta=1$ for the exact case in $1 D$ and the likely case in 2D. Thus, our results strongly suggest that the Heisenberg antiferromagnet on the $2 \mathrm{D}$ square lattice possesses non-vanishing long-range order. This agrees with other calculations for this case. Thus, for example, SWT at $\Delta=1$ gives $^{32} \mathrm{M}_{\mathrm{c}}^{2} \approx 0.606$ (compared to a divergent result, $\mathrm{M}^{2} \rightarrow-\infty$ as $\Delta \rightarrow 1$, in 1D), while the GFMC results ${ }^{30}$ of Carlson and of Trivedi and Ceperley give respectively $\mathrm{M}_{\mathrm{c}}^{\mathrm{z}} \approx 0.68 \pm 0.02,0.62 \pm 0.04$ at the same point. 


\section{CCM PARAMETRIZATION OF EXCITED STATES}

Within the CCM excited-state wavefunctions are written in the standard ${ }^{33}$ form, $\left|\Psi_{e}\right\rangle=X|\Psi\rangle=X e^{S}|\Phi\rangle$, in terms of an excitation operator $X$ which is chośen, like $S$, to be constructed purely from creation operators. By combining the g.s. Schrödinger equation (6) with its excited-state counterpart, $H\left|\Psi_{e}\right\rangle=E_{e}|\Psi\rangle$, we readily obtain

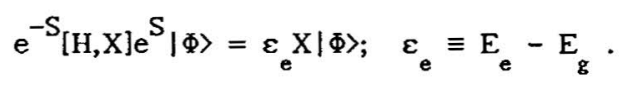

The simplest possible form for $X$ which produces excitations with $s_{z}^{\text {total }}= \pm 1$ has the form

$$
\mathrm{x} \rightarrow \mathrm{x}_{1}=\sum_{\mathrm{i}} \chi_{\mathrm{i}} \sigma_{\mathrm{i}}^{+}
$$

where the sum on $\mathbf{i}$ runs over one sublattice only, as before. The coefficients $\left\{\chi_{i}\right\}$ are obtained in the by now familiar manner by taking the overlap of Eq. (29) with the bra states $\langle\Phi| \sigma_{k^{-}}^{-}$If we combine the approximation of Eq. (30) for $\mathrm{X}$ with the SUB2 approximation of Eq. (14) for S, we obtain the set of homogeneous linear equations,

$$
\frac{1}{2} \mathrm{zK} \chi_{k}-\frac{1}{2} \sum_{\rho} \sum_{r} b_{r} \chi_{k-r+\rho}=\varepsilon_{e} \chi_{k} \text {, }
$$

which are valid for a bipartite lattice of arbitrary dimensionality, and where indices $\{r\}$ and $\{\rho\}$ have their earlier meanings.

It is trivial to verify that Eq. (31) has lattice plane-wave solutions,

$$
\chi_{k} \rightarrow \underset{\vec{k}}{\chi}(\overrightarrow{\mathrm{q}})=\exp (-\mathrm{i} \overrightarrow{\mathrm{k}} \cdot \overrightarrow{\mathrm{q}}) \chi(\overrightarrow{\mathrm{q}}) ; \quad \varepsilon \varepsilon_{\mathrm{e}} \rightarrow \varepsilon(\overrightarrow{\mathrm{q}})
$$

By making further use of the g.s. SUB2 solution of Eq. (20) and its counterpart for higher dimensionality, we obtain the spectrum,

$$
\varepsilon(\overrightarrow{\mathrm{q}})=\frac{1}{2} \mathrm{zK}\left[1-\mathrm{k}^{2} \gamma^{2}(\overrightarrow{\mathrm{q}})\right]^{\frac{1}{2}} \text {, }
$$

where the constants $\mathrm{K}$ and $\mathrm{k}^{2}$ are as given in Eqs. (16) and (21), and

$$
\gamma(\vec{q}) \equiv \frac{1}{z} \sum_{\vec{p}} \mathrm{e}^{\mathrm{i} \vec{p} \cdot \vec{q}}
$$

The spectrum of Eq. (33) thus has a finite gap in both $1 \mathrm{D}$ and $2 \mathrm{D}$ for the case $\mathrm{k}^{2}\langle 1$, or equivalently $\Delta\rangle \Delta_{\mathrm{c}}$. The gap disappears precisely at the critical value $\Delta_{c}$, again exactly as expected at a phase transition. In $1 \mathrm{D}$, we find that as $\Delta \rightarrow \Delta_{c}, \varepsilon(\mathrm{q}) \rightarrow \varepsilon_{\mathrm{c}}(\mathrm{q}) \approx 1.364|\sin (\mathrm{q})|,-\pi<\mathrm{q} \leq \pi$, which may be compared with the exact result ${ }^{25}$ at $\Delta=1$, namely $\varepsilon(q)=\frac{1}{2} \pi|\sin (q)|$. Similarly, for the 2D square lattice at $\Delta=\Delta_{c}$ we have the gapless spectrum,

$$
\varepsilon_{c}(\vec{q})=2 K_{c}\left[1-\frac{1}{4}\left(\cos q_{x}+\cos q_{y}\right)^{2}\right]^{\frac{1}{2}} ; \quad-\pi<q_{x}, q_{y} \leq \pi,
$$

with spin-wave velocity $v_{s}=2 K_{c} \approx 2.335$. This may be compared with the 
classical value $v_{0}=2$ at $\Delta=1$, and with the corresponding results for the Heisenberg square lattice of $2.28 \pm 0.1$ by Trivedi and Ceperley ${ }^{30}$ using GFMC techniques, and 2.316 by Oguchi from Anderson's SWT, ${ }^{32}$ evaluating the first-order correction in inverse powers of the spin quantum number.

\section{EXTENSION TO SYSTEMS WITH HIGHER SPIN}

All of the results presented so far have been for spin- $\frac{1}{2}$ systems. Unlike many other methods, the CCM has no problem in principle or in practice to be extended to systems of higher spin, $s>\frac{1}{2}$. The main changes are that we can no longer use the Pauli matrices, and the raising operator $s_{k}^{+}$(defined in the Néel basis as before) can now act up to 2 s times on the same site $k$. Thus,

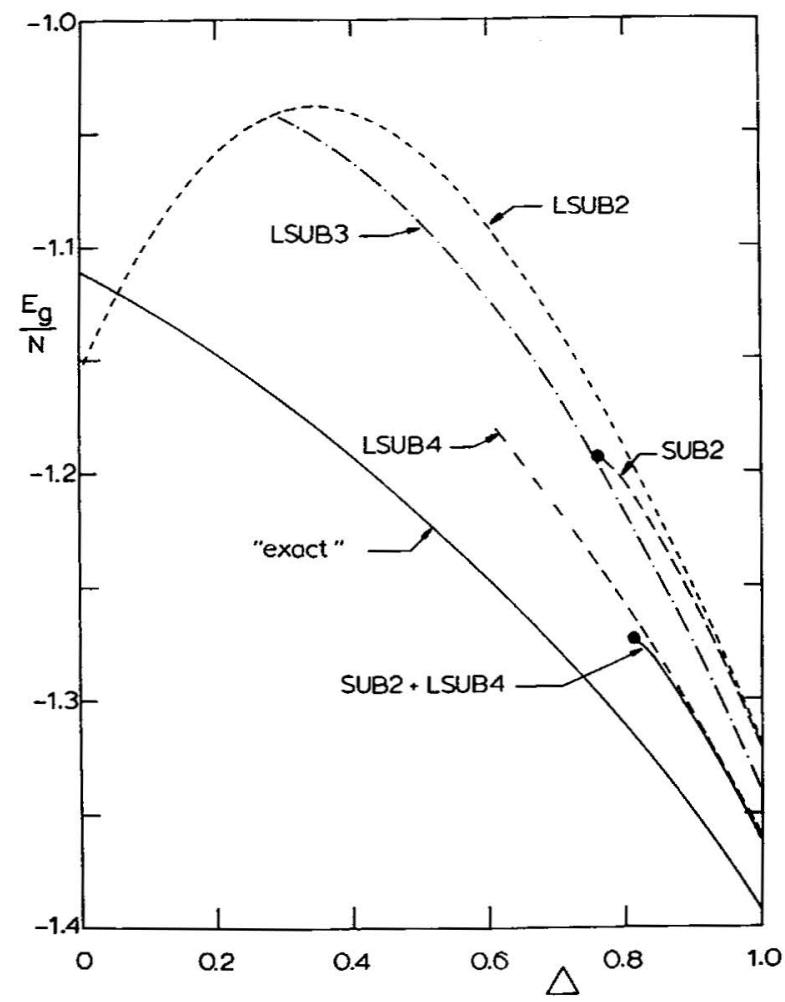

Fig. 6. Ground-state energy per spin for the spin-1 XXZ model in ID as a function of anisotropy parameter $\Delta . \quad$ CCM results in various approximations are compared with "exact" results obtained by us as described in the text.

for example, the cluster configuration coefficients in Eq. (13) can now have a given lattice index occurring up to $2 s$ times. Nevertheless, the basic implementation of the CCM remains unchanged, apart from the consequent increase in the number of independent cluster configurations at a given truncation level. For example, on the 1D chain, whereas in the spin- $\frac{1}{2}$ case the LSUB2 and LSUB4 approximations retained 1 and 3 independent configurations respectively, for the spin-1 case these numbers increase to 2 and 7 respectively. 
Typical results for the g.s. energy of the spin-1 chain, for example, are shown in Fig. 6 and Table 3 for a number of CCM approximation schemes described previously. Exact results are not known for the spin-1 XXZ model in $1 D$, but we compare with results extrapolated to the infinite $(N \rightarrow \infty)$ chain from exact diagonalizations that we have performed on finite chains of length $\mathrm{N} \leq 14$. We also compare with results from Anderson's linear spin-wave theory (SWT). ${ }^{32}$ As expected, the quantum fluctuations now play a relatively less important role in the Ising-phase region for higher values of the spin quantum number (as also is the case for higher dimensionality lattices). For example, in the SUB2 and SUB2+LSUB4 approximations, the critical values, $\Delta_{c} \approx 0.759$ and 0.812 respectively, are appreciably closer to the classical value of 1 than their counterparts for the spin- $\frac{1}{2}$ case in Sec. 4.

Table 3. The g.s. energy per spin, $E_{g} / N$, for the spin-1 Heisenberg model on an infinite $(\mathrm{N} \rightarrow \infty)$ chain. Results in various CCM schemes are compared with "exact" results extrapolated to $N \rightarrow \infty$ from exact diagonalizations on chains of length $N \leq 14$, and with results from Anderson's linear spin-wave theory (SWT).

\begin{tabular}{llllllll}
\hline \multicolumn{7}{c}{$\mathrm{E}_{\mathbf{g}} / \mathrm{N}$} \\
\cline { 2 - 8 } & LSUB2 & LSUB3 & LSUSB4 & SUB2 & $\begin{array}{c}\text { SUB2+ } \\
\text { LSUB4 }\end{array}$ & SWT & "Exact" \\
\hline 0.5 & -1.0594 & -1.0893 & -1.1278 & -- & -- & -- & -1.224 \\
1.0 & -1.3206 & -1.3404 & -1.3619 & -1.3224 & -1.3628 & -1.3634 & -1.398 \\
1.5 & -1.7189 & -1.7273 & -1.7329 & -1.7178 & -1.7329 & -1.6840 & -1.735 \\
2.0 & -2.1653 & -2.1694 & -2.1715 & -2.1646 & -2.1715 & -2.1316 & -2.172 \\
\hline
\end{tabular}

We note that the SUB2 approximation takes only independent 2-body correlations into account. Hence, it cannot be expected to distinguish qualitative differences between the $s=\frac{1}{2}$ and $s>\frac{1}{2}$ models. The interesting open question as to whether the $s=1$ case shows a qualitatively different phase structure in $\Delta$ than the $s=\frac{1}{2}$ case, as expected by the Haldane conjecture, ${ }^{1}$ could only properly begin to be addressed within the CCM at the SUB4 level. Such full SUB4 calculations have not yet been performed.

\section{DISCUSSION}

We believe that the results presented in this paper clearly demonstrate the viability of the CCM to deal accurately, and at a fully microscopic level, with the quantum fluctuations of discrete spin-lattice models. The method presupposes no prior knowledge of any phase transitions. Nevertheless, rather low-level approximation schemes seem quite capable of shedding light on such global properties, as well as giving accurate numerical results for such quantities as the g.s. energy and the staggered magnetization. Excitation spectra are also capable of being calculated. We have shown how various hierarchies of approximation schemes can be exploited to yield sets of results of increasing accuracy, by the systematic inclusion of higher-order 
correlations. We are currently pursuing such schemes as the LSUBn hierarchy to higher values of the truncation index $\mathrm{n}$, using computer-algebraic methods to derive the corresponding coupled sets of equations, in order to examine the convergence properties.

We have shown explicitly how, unlike many other methods, the CCM can be applied to bipartite spin lattices of arbitrary spin and dimensionality. Further work in progress on the spin-1 chain (using the SUB4 approximation, for example) may enable us to discuss the so-called Haldane phase. ${ }^{1}$ We are also interested both in extending our calculations on the present systems to include the effects of an external magnetic field, and in enlarging the class of model Hamiltonians. An interesting example for spin-1 systems is one which includes an arbitrary admixture of isotropic Heisenberg (quadratic) and biquadratic exchange terms. Frustrated systems are another example. Finally, we are also interested in extending our methodology to such comparable models as the $t-J$ and Hubbard models, as well as to such lattice gauge field theories as the $\mathrm{U}(1)_{3}$ model of quantum electrodynamics on a square lattice.

\section{ACKNOWLEDGEMENT}

One of us (RFB) gratefully acknowledges support for this work in the form of a research grant from the Science and Engineering Research Council (SERC) of Great Britain.

\section{REFERENCES}

1. F.D.M. Haldane, Phys. Lett. 93A:464 (1983); Phys. Rev. Lett. 50:1153 (1983); I. Affleck, J. Phys.: Condens. Matter 1:3047 (1989).

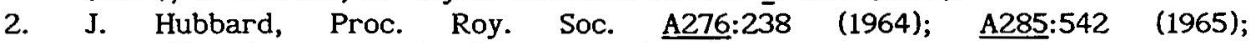
D. Vollhardt, Rev. Mod. Phys. 56:99 (1984).

3. P.W. Anderson, Science 235:1196 (1987).

4. D. Vaknin et al., Phys. Rev. Lett. 58:2802 (1987); G. Shirane et al., ibid. 59:1613 (1987); K.B. Lyons et al., ibid. 60:732 (1988).

5. R.F. Bishop and H.G. Kümmel, Phys. Today $40(3): 52$ (1987).

6. F. Coester, Nucl. Phys. 7:421 (1958); F. Coester and H. Kümmel, ibid. 17:477 (1960).

7. J. Paldus, J. Cižek and I. Shavitt, Phys. Rev. A $\underline{5}: 50$ (1972).

8. R.F. Bishop and K.H. Lührmann, Phys. Rev. B 17:3757 (1978).

9. H. Kümmel, K.H. Lührmann and J.G. Zabolitzky, Phys. Rep. 36C:1 (1978).

10. J.S. Arponen, Ann. Phys. (N.Y.) 151:311 (1983).

11. J. Arponen, R.F. Bishop and E. Pajanne, Phys. Rev. A 36:2519,2539 (1987); in: "Condensed Matter Theories," Vol. 2, P. Vashishta, R.K. Kalia and R.F. Bishop (eds.), Plenum, New York (1987), p. 357.

12. R.J. Bartlett, J. Phys. Chem. 93:1697 (1989).

13. R.F. Bishop, Theor. Chim. Acta (1991) - to be published.

14. H.G. Kümmel, in: "Nucleon-Nucleon Interaction and Nuclear Many-Body Problems," S.S. Wu and T.T.S. Kuo (eds.), World Scientific, Singapore (1984), p. 46.

15. R.F. Bishop et al., Phys. Rev. C 42:1341 (1990).

16. B. Day, Phys. Rev. Lett. 47:226 (1981); B. Day and J.G. Zabolitzky, Nucl. Phys. A366:221 (1981).

17. R.J. Bartlett, Ann. Rev. Phys. Chem. 32:359 (1981); K. Szalewicz et al., J. Chem. Phys. 81:2723 (1984); A.C. Scheiner et al., ibid. 87:5361 (1987); E.A. Salter, G.W. Trucks and R.J. Bartlett, ibid. 90:1752 (1989).

18. R.F. Bishop and K.H. Lührmann, Phys. Rev. B 26:5523 (1982); K. Emrich and

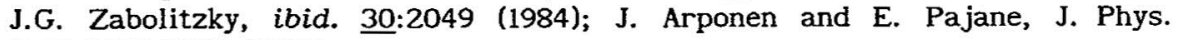
C $15: 2665,2683$ (1982).

19. U. Kaulfuss and M. Altenbokum, Phys. Rev. D 33:3658 (1986); R.F. Bishop, M.C. Boscá and M.F. Flynn, Phys. Rev. A $\underline{40: 3484}$ (1989); J.S. Arponen and R.F. Bishop, Phys. Rev. Lett. 64:111 (1990). 
20. M. Altenbokum and H. Kümmel, Phys. Rev. D 32:2014 (1985); M. Funke, U. Kaulfuss and H. Kümmel, ibid. 35:621 (1987).

21. H.A. Bethe, Z. Phys. 71:205 (1931).

22. L. Hulthén, Arkiv Mat. Astron. Fysik A 26:No. 11 (1938); R. Orbach, Phys. Rev. 112:309 (1958).

23. R.J. Baxter, J. Phys. C $\underline{6}:$ L94 (1973); J. Stat. Phys. 9:145 (1973).

24. N.M. Bogoliubov, A.G. Izergin and V.E. Korepin, Nucl. Phys. B275:687 (1986).

25. J. des Cloiseaux and J.J. Pearson, Phys. Rev. 128:2131 (1962); C.N. Yang and C.P. Yang, ibid. 150:321 (1966); J.D. Johnson, S. Krinsky and B.M. McCoy, Phys. Rev. A $\underline{8}: 2526$ (1973); L.D. Faddeev and L.A. Takhtajan, Phys. Lett. 85A:375 (1981).

26. R.F. Bishop, J.B. Parkinson and Yang Xian, in: "Condensed Matter Theories," Vol. 6, S. Fantoni and S. Rosati (eds.), Plenum, New York (1991), p. 37; Phys. Rev. B. (1991) -- to be published.

27. M. Roger and J.H. Hetherington, Phys. Rev. B 41:200 (1990).

28. R.F. Bishop, J.B. Parkinson and Yang Xian, Phys. Rev. B $\underline{43}: 13782$ (1991).

29. S. Sachdev, Phys. Rev. B 39:12232 (1989).

30. J. Carlson, Phys. Rev. B $\underline{40: 846 ~(1989) ; ~ N . ~ T r i v e d i ~ a n d ~ D . M . ~ C e p e r l e y, ~}$ ibid. $41: 4552$ (1990).

31. T. Barnes, D. Kotchan and E.S. Swanson, Phys. Rev. B 39:4357 (1989).

32. P.W. Anderson, Phys. Rev. 86:694 (1952); T. Oguchi, Phys. Rev. 117:117 (1960).

33. K. Emrich, Nucl. Phys. A351:379,397 (1981). 